\title{
Germanica
}

\section{Réflexions sur quelques formes de l'altérité dans les nouvelles de jeunesse de Thomas Mann}

On certain forms of alterity in Thomas Mann's early short stories

Überlegungen zu Formen der Alterität in Thomas Manns frühen Erzählungen

\section{Éric Leroy du Cardonnoy}

\section{(2) OpenEdition}

\section{Journals}

Édition électronique

URL : http://journals.openedition.org/germanica/3772

DOI : ERREUR PDO dans /localdata/www-bin/Core/Core/Db/Db.class.php L.34 : SQLSTATE[HYO00]

[2006] MySQL server has gone away

ISSN : 2107-0784

Éditeur

Université de Lille

\section{Édition imprimée}

Date de publication : 30 juin 2017

Pagination : 153-165

ISBN : 9782913857391

ISSN : 0984-2632

Référence électronique

Éric Leroy du Cardonnoy, «Réflexions sur quelques formes de l'altérité dans les nouvelles de jeunesse de Thomas Mann », Germanica [En ligne], 60 | 2017, mis en ligne le 30 juin 2019, consulté le 08 janvier 2021. URL : http://journals.openedition.org/germanica/3772 ; DOI : https://doi.org/10.4000/ germanica.3772 


\title{
Réflexions sur quelques formes de l'altérité dans les nouvelles de jeunesse de Thomas Mann
}

\author{
Éric LEROY DU CARDONNOY \\ Université Caen-Normandie ${ }^{1}$
}

La question de l'altérité pourrait a priori sembler aller de soi en ce qui concerne les nouvelles de jeunesse de Thomas Mann; elle est importante, sans être centrale, au sens où l'écrivain la prendrait comme sujet principal de ses nouvelles ; il peut s'agir de l'altérité sexuelle, physique, sociale, géographique, raciale, voire culturelle - nous ne pourrons pas ici traiter toutes ces formes de l'altérité, et nous ne nous concentrerons que sur quelques-unes, qui nous semblent saillantes. Cependant, du fait de sa biographie personnelle, la question n'est pas sans influence sur l'écriture et la vision du monde que présente Thomas Mann. Nous voudrions donc, de manière, bien entendu tout à fait succincte, mettre en avant quelques éléments qui pourraient former les prémices d'une réflexion sur le sujet.

La première figure de l'altérité qui nous intéressera, est celle du tout autre, de celui, ou celle, qui se trouve aux antipodes des sujets narrateurs. Ainsi, le monde colonial est très présent dans les nouvelles de jeunesse, les rapports entre la métropole et les colonies ou bien les liens entre les « maîtres » et les colonisés apparaissent en filigrane de nombreux

1. - Normandie Univ. Caen, ERLIS EA 4254.

GERMANICA, 2017, LX, pp. 153 à 165. 
textes. Comme le rappellent Friedrichsmeyer, Lennox et Zantop dans l'introduction à leur recueil ${ }^{2}$, même si la période coloniale de l'Allemagne n'a duré que de 1884 à 1919, la question du colonialisme n'en est pas moins pertinente que dans les pays qui ont eu plus tôt et plus longtemps un empire colonial, et même si les territoires d'outre-mer n'ont pas été aussi profitables économiquement que ceux des Britanniques ou des Français par exemple. Mais cet empire colonial a tout de même eu une importance historique et culturelle, pour l'Allemagne et pour ses colonies. Contrairement cependant aux deux derniers exemples, il n'existe pratiquement pas de littérature postcoloniale en allemand par les descendants des colonisés ou par les colons eux-mêmes ${ }^{3}$, même s'il existe une germanistique post-coloniale. Il ne semble pas qu'il y ait, comme l'affirme Homi Bhabha pour d'autres empires coloniaux, des « personnes en mouvement qui ne peuvent être contenues dans les limites du Heim de la culture nationale et du discours univoque qu'elle produit », menaçant ainsi « les frontières de la nation moderne » 4 , ce qui reste à voir. Mais il est toutefois possible d'affirmer que, même s'il n'y a pas de problématique de type postcolonial sur le modèle existant pour les États-Unis, la Grande-Bretagne, la France, l'Espagne, le Portugal ou même l'Italie, la question est loin de pouvoir être aussi simplement évacuée. Ce n'est pas ici le lieu de s'interroger sur les raisons de ces différences, mais plutôt de prendre le cas de Thomas Mann et de ses nouvelles de jeunesse ${ }^{5}$ comme un exemple parmi d'autres ; les inter-

2. - Sara Friedrichsmeyer, Sara Lennox, Susanne Zantop (dir.), The Imperialist Imagination: German Colonialism and Its Legacy, Ann Harbor University of Michigan Press, 1998 (Series: Social history, popular culture, and politics in German), p. 3. Les nouvelles de Thomas Mann seront citées d'après : Thomas Mann, Frühe Erzählungen 1893-1912 in der Fassung der Großen kommentierten Frankfurter Ausgabe, Frankfurt a. M., Fischer Taschenbuch Verlag, 2014 (1. Auflage 2008), Fischer Klassik Nr. 90405 et, sauf indication, pour la traduction française Thomas Mann, Romans et nouvelles I. 1896-1903, préf. de Claude David ; notices et notes de Claude David et Armand Nivelle ; trad. de Félix Bertaux, Geneviève Bianquis, Geneviève Maury et al., Paris, Librairie générale française, 1994, «La pochothèque. Classiques modernes », et Romans et nouvelles II. 1904-1924, notices et notes de Jean-Louis Bandet et Jean-Marie Valentin ; trad. de Félix Bertaux, Maurice Betz, Louise Servicen et al., Paris, Librairie générale française, 1995, « La pochothèque. Classiques modernes ».

3. - On pourra citer ici Giselher W. Hoffmann comme exemple de littérature de langue allemande de Namibie.

4. - Homi K. Bhabha, « DissemiNation: Time, narrative and the margins of the modern nation », in : The Location of Culture, New York, Routledge, 1994, p. 139-170, ici p. 164 : « wandering peoples who will not be contained within the Heim of the national culture and its unisonant discourse, but are themselves the marks of a shifting boundary that alienates the frontiers of the modern nation. »

5. - Nous ne considérerons dans cet article que les œuvres de jeunesse de Thomas Mann, tout en nous réservant la possibilité de faire référence à d'autres œuvres de la même époque et notamment ses romans, qui seraient plus à même d'étayer notre argu- 
rogations de type postcolonial sont bien présentes, même si c'est de manière oblique, voire indirecte et sous-jacente ${ }^{6}$. Nous verrons ainsi que les raisons autobiographiques, tout simplement, ont permis à l'écrivain de s'interroger sur la vision dominante, allemande, de la culture (master narrative), qu'il n'hésitera d'ailleurs pas à défendre en 1918 contre les puissances alliées dans ses Considérations d'un apolitique. Les relations entre colonisateurs et colonisés sont déportées vers une restructuration, propre à Thomas Mann, de l'antagonisme métropole-colonisés pour la période allant jusque 1912, à savoir les relations entre le nord et le $\operatorname{sud}^{7}$, entre ce que l'on pourrait nommer le principe masculin et le principe féminin, qu'il met en scène en des termes qui peuvent faire songer à des interrogations postcoloniales. Mais dans le même temps, il est aussi victime du discours national dominant, auquel il ne parvient pas à échapper totalement, notamment en ce qui concerne la supériorité des Allemands sur les autres peuples, européens ou extra-européens.

C'est pourquoi nous verrons dans un premier temps quelquesunes des formes sous lesquelles l'altérité apparaît dans les nouvelles de jeunesse, puis nous nous attacherons à ses modes de re-présentation significatifs afin de comprendre de quelle manière Thomas Mann intègre la dichotomie Europe-mondes extra-européens dans une vision de la décadence fin de siècle.

Le monde « colonial » peut apparaître dans un tout premier temps comme quelque chose de purement décoratif, correspondant en cela à la mode de la fin du XIX ${ }^{\mathrm{e}}$ siècle en Europe occidentale : il s'agit ainsi par exemple, dans La Chute (Gefallen), de l'atelier-salon de Meysenberg décoré de vases japonais, d'éventails chinois, de cornes d'écailles d'Afrique $^{8}$ ou du salon d'Amra dans Louisette (Luischen) où l'on trouve une ottomane, un palmier et une table en acajou ${ }^{9}$, et la salle de fête est

mentation.

6. - Voir par ex. Yahya A. Elsaghe, Die imaginäre Nation: Thomas Mann und das «Deutsche », München, Fink, 2000 et Todd Curtis Kontje, Thomas Mann's World: Empire, Race, and the Jewish Question, Ann Arbor, University of Michigan Press, 2011.

7. - Nous restons pour l'instant relativement vague en ce qui concerne ces deux notions que nous allons préciser par la suite. Qu'il soit tout de même permis de mentionner dès maintenant qu'elles sont à entendre à la fois comme intra-, mais aussi extraeuropéennes.

8. - Thomas Mann, Mario et le magicien suivi de Expériences occultes et autres récits (trad. André Gailliard et Louise Servicen), Paris, Grasset, 1973, La Chute, p. 153 / Gefallen: «Das war ein seltsamer Raum, hergerichtet in einem einzigen Stile: bizarre Künstlerlaune. Etrurische und japanesische Vasen, spanische Fächer und Dolche, chinesische Schirme und italienische Mandolinen, afrikanische Muschelhörner [...]», p. 14.

9. - Romans et nouvelles I, Louisette, p. 101/Luischen : " in Amras Salon [...] mit einer Ottomane nebst vielen Kissen, einer Fächerpalme, englischen Ledersesseln und einem Mahagoni-Tisch », p. 168. 
bâtie « dans un drôle de salmigondis de style chinois et Renaissance »10, qui font songer par exemple à des photos prises à peu près à la même époque de Sarah Bernhardt chez elle ou encore de Karl May dans le bureau de sa villa à Radebeul. Dans Paillasse (Der Bajazzo), une fête de charité - le texte allemand utilise le terme «Bazar » avec les guillemets afin de souligner le côté exotique de la chose -, est organisée par une œuvre de bienfaisance de la ville; l'utilisation des guillemets dans le texte original souligne immédiatement la distance et le côté exotique du mot qui sont repris par des termes associés à l'exotisme11 ou par les costumes que les femmes qui tiennent les stands ont revêtus - elles sont habillées en Italiennes.

Mais le monde colonial apparaît également dans sa fonction décorative à un autre niveau : ainsi dans Louisette, Amra décide d'organiser comme l'un des numéros de sa fête des danses nègres 12 afin de distraire ses invités, et dans L'Enfant prodige (Das Wunderkind) le lieutenant Adolf se met « à exécuter sur la neige durcie par le gel une petite danse nègre, parce qu'il fait si froid. »13 $\mathrm{Il}$ y a là une fonction de divertissement dévolue au monde colonial, autant pour le conjurer que pour s'en distancer dans un sentiment de supériorité culturelle.

L'Autre colonial est aussi présent en filigrane, en creux pourrait-on dire, dans quelques nouvelles, du fait que les pères des personnages principaux sont des hommes d'affaires, dans des villes portuaires du Nord de l'Allemagne, et que leur commerce, comme pour les Buddenbrook, concerne les liens et les échanges avec les territoires des colonies. Que ce soit le père de Paolo Hofmann, riche planteur d'Amérique du Sud, ou les géniteurs de Tonio Kröger et Hans Hansen - le premier dans le commerce du grain, le second dans le commerce du bois (pour les navires probablement ou les tonneaux de transport) ${ }^{14}$.

10. - Ibid., p. 105/Ibid. : « in einem drolligen Stilgemisch aus Chinesisch und Renaissance erbaut », p. 174.

11. - Romans et nouvelles I, Paillasse, p. 71/Der Bajazzo : «schwüler Dunst von Wein, Speisen, Parfüms und Tannengeruch, ein wirrer Lärm von Gelächter, Gespräch, Musik, Ausrufen und Gongschlägen entgegendrang. », p. 153.

12. - Romans et nouvelles I, Louisette, p. 107/Luischen : «Studenten, die durch den Assessor eingeführt worden waren und Negertänze zur Aufführung bringen sollten », p. 168.

13. - Thomas Mann, Tonio Kröger (trad. Geneviève Maury), Paris, Stock, 2004 («La Cosmopolite »), L'Enfant prodige, p. 215/Das Wunderkind : « [Lieutenant Adolf] fängt [...] im Gehen ein bischen an, auszuschlagen, mit emporgeklapptem Kragen und die Hände in den schrägen Manteltaschen auf dem hartgefrorenen Schnee einen kleinen nigger-dance aufzuführen. [...]. », p. 406.

14. - Romans et nouvelles I, Tonio Kröger, p. 210/Tonio Kröger « die weitläufigen Holz-Lagerplätze drunten am Fluß, wo gewaltige Sägemaschinen unter Fauchen und Zischen die Stämme zerlegten. Aber Tonio war Konsul Krögers Sohn, dessen Getreidesäcke mit dem breiten Firmendruck man Tag für Tag durch die Straßen 
Rappelons ici quelques éléments biographiques concernant les origines de Thomas Mann lui-même. On le sait, sa mère, Julia da SilvaBruhns (1851-1923) était originaire du Brésil, fille d'un planteur né à Lübeck et émigré au Brésil qui avait épousé l'héritière d'un grand propriétaire terrien portugais. Autrement dit, le monde colonial est présent dans les origines et les racines mêmes de Thomas Mann et ne cessera de l'occuper.

Cette alliance de son aïeul et de son père se retrouve dans plusieurs nouvelles de jeunesse et fonde l'identité de plusieurs personnages ; Thomas Mann intègre de la sorte des éléments de sa propre biographie à sa fiction narrative et à la biographie de nombre de ses personnages, un seul exemple suffira ici :

Le vieux Hofmann avait gagné sa fortune comme planteur en Amérique du Sud. Il y avait épousé une indigène de bonne famille et était bientôt rentré avec elle en Allemagne du Nord, sa patrie. Ils vivaient dans ma ville natale où résidait également le reste de sa famille. Paolo y était né15.

Tels sont par exemple les premiers mots de la nouvelle La Volonté de bonheur (Der Wille zum Glück). Dans la nouvelle La Mort (Der Tod), la fille du comte, Asuncion, a une mère originaire de Lisbonne ${ }^{16}$, mais qui est déjà décédée. Cette identité non allemande, voire non européenne, est le cas également de la mère de Tonio Kröger, Consuelo :

Quant à la mère de Tonio, sa belle maman aux cheveux noirs qui portait le prénom de Consuelo et ressemblait si peu aux autres dames de la ville, parce que le père avait été la chercher jadis tout au bas du planisphère, les bulletins lui étaient totalement indifférents ${ }^{17}$.

Même si le continent n'est pas précisé, on est en droit de suspecter qu'il s'agit de l'Amérique du Sud. La formulation est intéressante aussi

$\overline{\text { kutschieren sah », p. }} 244$.

15. - Thomas Mann, Mario et le magicien..., op. cit., La Volonté de bonheur, p. 199/Der Wille zum Glück: «Der alte Hofmann hatte sein Geld als Plantagenbesitzer in Südamerika verdient. Er hatte dort eine Eingeborene aus gutem Hause geheiratet und war bald darauf mit ihr nach Norddeutschland, seiner Heimat, gezogen. Sie lebten in meiner Vaterstadt, wo auch seine übrige Familie zu Hause war. Paolo wurde hier geboren. » p. 50. p. 73.

16. - Thomas Mann, Mario et le magicien..., op. cit., La Mort, p. 227/Der Tod,

17. - Romans et nouvelles I, Tonio Kröger, p. 212/Tonio Kröger : « Der Mutter Tonios jedoch, seiner schönen, schwarzhaarigen Mutter, die Consuelo mit Vornamen hieß und überhaupt so anders war, als die übrigen Damen der Stadt, weil der Vater sie sich einstmals von ganz unten auf der Landkarte heraufgeholt hatte, - seiner Mutter waren die Zeugnisse grundeinerlei... », p. 247. 
à un autre niveau : en effet le père est allé la chercher « tout en bas du planisphère » (« heraufgeholt » en allemand) ; cela signifie que la mère est comme un objet exotique que l'on va chercher et rapporte d'un voyage. De plus, la formulation se double d'une référence, qui n'est pas que spatiale : «heraufholen » implique un mouvement du bas vers le haut, comme si seul ce qui est en haut était digne, supérieur. La cartographie, faite par les Européens, est donc normée par et pour les Européens avec le « Nord » du globe terrestre en position supérieure. Ainsi, le continent européen est celui qui est capable de conférer une « existence » réelle, en divisant le monde selon des repères axiologiques ; ce qui est noneuropéen est regardé de haut précisément, comme inférieur («minderwertig »), dans une position subalterne. De plus, la mère de Tonio est un être musical, que le succès ou la rentabilité n'intéressent pas, à la différence du père commerçant. Les prénoms de plusieurs personnages masculins et féminins, comme ceux déjà cités, trahissent les origines non-européennes des personnages comme Amra ${ }^{18}$ dans Louisette, Tonio dans Tonio Kröger, Bibi Saccellaphyllacas dans L'Enfant prodige, Do Escobar ${ }^{19}$ dans Comment Jappe et Do Escobar se battirent (Wie Jappe und Do Escobar sich prügelten).

Même sil'intégration parThomas Mann d'éléments autobiographiques à sa fiction narrative est intéressante en soi, elle révèle également une construction particulière, qui établit deux sphères différentes et antagonistes, fait de l'opposition entre l'Europe, l'Allemagne et le reste du monde - latin dans sa grande majorité - non européen un critère d'appréciation de la situation et de la position sociale des personnages ; on peut faire l'hypothèse que leur relation fonctionne comme une opposition structurale et structurante, et l'hybridité ne fonctionne pas comme un facteur positif, au contraire. Un seul exemple suffira à exemplifier cette situation :

« Oui, c'est un nom stupide, Dieu sait que j'aimerais mieux m'appeler Henri ou Guillaume, vous pouvez m'en croire ! Mais j'ai été appelé ainsi d'après un frère de ma mère qui s'appelle Antonio ; car ma mère n'est pas d'ici, comme vous le savez... » [...].

Mais « Tonio » avait quelque chose d'étranger et de singulier. Oui, il avait quelque chose de singulier en lui sous tous les rapports, qu'il le voulût ou non, et il était seul et exclu du milieu des gens comme il faut

18. - Nous reviendrons plus loin sur cet exemple, unique dans le corpus qui est le nôtre : en effet, il s'agit d'un prénom choisi volontairement par le personnage et qui n'a aucun lien avec ses véritables origines.

19. - Romans et nouvelles II, Comment Jappe et Do Escobar se battirent, p. 81/ Wie Jappe und Do Escobar sich prügelten : « Do Escobar war von freier Natur, ein exotischer Fremdling, der nicht einmal regelmäßig zur Schule ging, sondern nur hospitierte und zuhörte (ein unordentliches, aber paradiesisches Dasein !) », p. 485. 
et habituels, bien qu'il ne fût pourtant pas un bohémien dans une roulotte verte, mais le fils du consul Kröger, de la famille des Kröger. Mais pourquoi Hans l'appelait-il Tonio tant qu'ils étaient seuls, et avait-il honte de lui dès qu'un troisième survenait ? [...] Mais lorsque Jimmerthal était arrivé, il avait tout de même poussé un soupir de soulagement, il l'avait délaissé, et il lui avait reproché sans nécessité son prénom étranger. Comme c'était douloureux de voir clair dans tout cela !... Hans Hansen l'aimait un peu au fond, quand ils étaient entre eux, Tonio le savait. Mais si un troisième survenait, Hans avait honte de lui et le sacrifiait ${ }^{20}$.

L'hybridité est ici discriminante - Tonio préférerait d'ailleurs, comme il l'avoue, s'appeler « Heinrich » ou «Wilhelm »- et la manière dont Tonio introduit ses remarques, bien involontaire sans doute, mais profondément révélatrice, insiste sur cet état de fait : il fait précéder chacune de ses remarques par un « aber »- ou ajoute un « doch »- à la fonction adversative, signalant par là même une contradiction fondamentale, qui résulte d'une évaluation des faits de l'ordre de la restriction, dans l'attitude de son ami d'une part, mais plus généralement dans le regard que la société wilhelminienne du Nord de l'Allemagne jette sur cet enfant, fruit d'un mariage que l'on appellerait aujourd'hui mixte. Le contact avec l'Autre « contamine » l'identité même de Hans qui n'est pas encore capable à son âge de mesurer les tenants et les aboutissants de la situation et de prendre clairement position en faveur de son ami. Une autre raison pour ce mépris de soi, dû à sa position hybride et « marginale », est celle qu'il livre lui-même par deux fois dans une identification, qu'il refuse, aux «tziganes à la voiture verte », opposant au nomadisme de l'autre la sédentarité de sa famille paternelle, garantie du droit à l'existence et à la stabilité selon lui.

Mais il n'y a pas que les lieux hors d'Europe qui peuvent être considérés, car les différents narrateurs et personnages regardent certaines parties du continent lui-même comme relevant d'une autre sphère que la leur et s'y comportent en « colonisateurs »; ainsi certains lieux euro-

20. - Romans et nouvelles I, Tonio Kröger, p. 216/Tonio Kröger : «,Ja, es ist ein alberner Name, ich möchte, weiß Gott, lieber Heinrich oder Wilhelm heißen, das könnt ihr mir glauben. Aber es kommt daher, daß ein Bruder meiner Mutter, nach dem ich getauft worden bin, Antonio heißt ; denn meine Mutter ist doch von drüben..." [...] Aber „Tonio“ war etwas Ausländisches und Besonderes. Ja, es war in allen Stücken etwas Besonderes mit ihm, ob er wollte oder nicht, und er war allein und ausgeschlossen von den Ordentlichen und Gewöhnlichen, obgleich er doch kein Zigeuner im grünen Wagen war, sondern ein Sohn Konsul Krögers, aus der Familie der Krögers... Aber warum nannte Hans ihn Tonio, solange sie allein waren, wenn er, kam ein dritter hinzu, anfing, sich seiner zuschämen ? [...] Aber als dann Jimmerthal gekommen war, hatte er dennoch erleichtert aufgeatmet, hatte ihn verlassen und ihm ohne Not seinen fremden Rufnamen vorgeworfen. Wie es weh that, dies alles durchschauen zu müssen! ... Hans Hansen hatte ihn im Grunde ein wenig gern, wenn sie unter sich waren, er wußte es. Aber kam ein dritter, so schämte er sich dessen und opferte ihn auf. », p. 252-253. 
péens sont assimilés à un monde « autre », relevant d'autres isotopies et de caractéristiques différentes : il s'agit principalement de l'Europe de l'Est et de l'Italie, comme le rappellent Yahya Elsaghe et Todd Curtis Kontje par exemple ${ }^{21}$. Ainsi dans les nouvelles La Volonté de bonheur (Der Wille zum Glück), Les Enfants de Wotan (Wälsungenblut), La Mort à Venise (Der Tod in Venedig) et Gladius Dei, l'Autre de l'Allemagne se trouve à la fois dans le Sud de l'Allemagne - à Munich ${ }^{22}$ - et de l'Europe en Autriche et en Italie mais aussi à l'Est, en Pologne. La posture des narrateurs est toujours de considérer l'Allemagne comme le centre et l'étalon des valeurs. Ainsi, l'Autriche-Hongrie devient un pays aux confins du continent où Orient et Occident se mêlent. Yahya Elsaghe prend l'exemple de La Mort à Venise et rappelle à quel point les origines d'Aschenbach, à la fois généalogiques et territoriales, sont hybrides et combien il réunit des caractéristiques antagoniques : il s'agit de l'opposition entre les origines du père et de la mère, de la différence sexuelle entre les deux qui se complètent par une opposition entre le côté cérébral et physique, entre le contrôle des instincts et la corporéité23. Ainsi toujours selon Yahya Elsaghe, l'Autriche-Hongrie marque une frontière, une délimitation entre la sphère allemande, masculine, patriarcale, symbole de l'ordre, de la santé et de la force physique et la sphère non-allemande, féminine, matriarcale, symbole de la sensibilité, de la maladie et de la sensualité. Vienne est ainsi la porte de l'Orient ${ }^{24}$ et de tous les fantasmes qui y sont attachés, fonction qui, dans la nouvelle, est reprise par Venise ${ }^{25}$. Cette ex-patriation d'Aschenbach et cette situa-

21. - Yahya A. Elsaghe, Die imaginäre Nation..., op. cit. et Todd Curtis Kontje, Thomas Mann's World..., op. cit.

22. - Comme le rappelle Yahya A. Elsaghe, il existe un tropisme munichois pour Thomas Mann et ses différents narrateurs (voir notamment Aschenbach) qui semble correspondre à cet appel du Sud, mais aussi à un autre rapport au corps et aux autres.

23. - Yahya A. Elsaghe, Die imaginäre Nation..., op. cit. : " An die Geschlechterdifferenz der Eltern lagert sich also der Gegensatz von 'Geistigkeit' und 'physische[r] Basis', von Triebkontrolle und Körperlichkeit an, wie ihn Thomas Mann nach Ausweis unzähliger Anstreichungen später bei seinen Bachofen-Lektüren in der Hierarchisierung der 'intellektuell'-'metaphysischen' Vater- und der 'stofflich'-'physischen' Mutterrechts-periode wieder- und wiederfinden sollte. », p. 38.

24. - Ibid., p. 42 : «porta Orientis [gilt] auch für jenen geheimnisvollen Orient, das Reich des Unbewußten ». " Das erinnert an Freuds eigene kolonialistische Metapher für die weibliche Sexualität, 'dark continent', und verrät die Homologie des psychoanalytischen und eines älteren, von Edward Said 'Orientalismus' genannten 'Diskurses' ». Voir aussi sur la question Hans Mayer, Außenseiter, Frankfurt a.M., Suhrkamp, 1975, S. 459 et pour la traduction par Laurent Mühleisen, Les Marginaux, Paris, Albin Michel, 1994 («Bibliothèque Albin Michel Idées »), p. 481.

25. - Yahya A. Elsaghe, Die imaginäre Nation..., op. cit., p. 43 : « Der Schnittpunkt von 'Nord' und 'Süd', 'Westen' und 'Osten' schlechthin. 'Venedig' liegt in einer imaginären Geographie, wie sie noch die postumen Interpreten des Texts bezeugen - so etwa Walter Jens und selbst Edward Said - : "an der Grenze von Europa 
tion extra-européenne de Venise vont lui permettre d'expérimenter ce qu'il n'a jamais pu faire auparavant ; Venise est ainsi le lieu où ce qui est interdit en «Europe » est possible, par exemple l'homosexualité (soumise aux interdits légaux du \$175 du Strafgesetzbuch), elle est aussi associée au côté dionysiaque - donc oriental ${ }^{26}$ - et par conséquent létal de la culture ${ }^{27}$. Aschenbach tombe amoureux de Tadzio, entre autres aussi parce qu'il appartient à cette sphère " orientale », aussi considérée comme inférieure culturellement et par conséquent exploitable, et n'est pas sans rappeler la découverte du corps des jeunes garçons orientaux par la photographie notamment vers la fin du XIX ${ }^{e}$ siècle - comme dans le cas de Gide, par exemple, lors de son voyage dans le Maghreb en 1893.

Hans Mayer, dans son ouvrage sur les marginaux, constate que les Lumières sont un échec en ce sens qu'elles ont postulé une égalité de nature entre les hommes, mais qu'il a bien vite fallu constater que ce n'était pas le cas en pratique. Ainsi s'est développée une logique de l'exclusion parce que d'une part, la position des Lumières étant une 《 conception globalisante ${ }^{28}$, il lui a été impossible de tenir compte du ou des cas particuliers en son sein même, et d'autre part parce que le capitalisme bourgeois des $\mathrm{XIX}^{\mathrm{e}}$ et $\mathrm{XX}^{\mathrm{e}}$ siècles a eu besoin pour fonctionner sur le principe de la concurrence économique de postuler l'inégalité - sur le plan économique, mais pas juridique. Cette contradiction a permis de générer toute une panoplie de formes et d'êtres incompatibles avec la morale bourgeoise et sa hiérarchie dont Hans Mayer dresse la phénoménologie : que ce soit d'un point de vue ontologique - ou pour reprendre les termes d'Hans Mayer « existentiel »- pour la femme, l'homosexuel ou le Juif, que ce soit d'un point de vue « intentionnel » pour les autres. Ainsi naît la catégorie du « marginal » qui ne peut s'intégrer en tant qu'Autre dans aucun groupe et ne peut former de communauté

und Asien", "at or just beyond the boundaries of Europe" ». Sur la même page, Elsaghe note que le narrateur attribue à l'architecture de Venise des traits arabes, que Tadzio vient des confins de l'Europe, à l'Est, même s'il n'est pas aussi barbare que les Russes qu'Aschenbach observe régulièrement. Enfin la mort qu'il subira vient de l'Orient : il s'agit du choléra « indien » et des effluves méphitiques du Gange (Der Tod in Venedig, p. 578) que les autorités tentent de cacher en vain et dont un Autrichien décèdera lui aussi après être rentré chez lui. Voir aussi Yahya A. Elsaghe, op. cit., p. 44 : « Venedig [wird] während der Erzählzeit immer mehr zur außereuropäischen, 'asiatischen' Stadt verfremdet. »

26. - Romans et nouvelles II, La Mort à Venise, p. 170/Der Tod in Venedig : «Die Liederlichkeit nahm aufdringliche und ausschweifende Formen an, wie sie sonst hier nicht bekannt und nur im Süden des Landes und im Orient zu Hause gewesen waren. », p. 580.

27. - Ibid., p. 160/Ibid. « Venedig, [...] halb Märchen, halb Fremdenfalle », p. 567.

28. - Hans Mayer, Les Marginaux, op. cit., p. 30 (traduction). 
véritable ${ }^{29}$, car le système capitaliste bourgeois saura toujours d'une manière ou d'une autre les récupérer ou les stigmatiser, que ce soit selon le modèle libéral, fasciste ou marxiste.

Le caractère non germanique de l'Autriche-Hongrie est rappelé dans d'autres nouvelles, dans lesquelles une vision germanocentrée apparaît très clairement. Ainsi, dans La volonté de bonheur, l'association à l'Autriche, dont est originaire le baron Stein, est teintée de relents très clairement antisémites :

On ne pouvait pas discerner avec certitude si l'anoblissement du baron n'avait pas été le résultat d'une perte de quelques syllabes de son nom ; par contre son épouse était tout simplement une vilaine petite juive dans une robe grise sans aucun goût. Â ses oreilles pendaient de grosses boucles étincelantes ${ }^{30}$.

L'Allemagne et sa culture sont considérées comme supérieures à tout point de vue 31 - il n'est que de regarder le portrait des nobles et aristocrates qui presque tous sans exception sont auréolés d'une force due à leur « race ${ }^{32}$, et l'opposition entre l'Allemagne et le reste du monde est, entre autres, déclinée sur le mode de la grande santé et de la pureté raciale, rappelant la fascination que Nietzsche a exercé sur le jeune Thomas Mann dont on sait qu'il a lu Au-delà du Bien et du Mal et Ainsi parlait Zarathoustra dans les années 1894-1895 selon ses cahiers ${ }^{33}$. L'antisémitisme est bien présent dans ces nouvelles de jeunesse, comme forme de réaction à la confrontation à ce qui est considéré comme " autre », et il est clair que Thomas Mann a par la suite changé sa manière de voir les choses, mais à cette époque il est sujet à

29. - Ibid., p. 86.

30. - Dans la traduction de La Volonté de bonheur par Louise Servicen, le passage consacré à la rencontre avec les parents d'Ada est malheureusement escamoté/Der Wille zum Glück : «Es ließ sich nicht mit Bestimmtheit erkennen, ob seiner Erhebung zum Freiherrn einst ein paar Silben seines Namens zum Opfer gefallen waren; dagegen war seine Gattin einfach eine häßliche kleine Jüdin in einem geschmacklosen grauen Kleid. An ihren Ohren funkelten große Brillanten. », p. 56-57.

31. - Cependant, d'autres nations sont aussi stigmatisées dans cette vision germano-centrée : les Français (dans Gerächt) par exemple, mais aussi les Russes, voire les Polonais (dans Mort à Venise).

32. - Il suffit de prendre les exemples de von Rinnlingen et sa femme dans Le Petit monsieur Friedemann (Der kleine Herr Friedemann), de la jeune femme et ses deux frères à la fin de L'Enfant prodige (p. 216/« Sie ist wunderschön mit ihren stahlblauen Augen und ihrem klaren, reinrassigen Gesicht, ein richtiges Edelfräulein », p. 406).

33. - Voir par exemple Daniela Langer «Imitation von Nietzsches Stil und imitatio Nietzsches - von der frühen Essayistik Thomas Manns bis zu den Betrachtungen eines Unpolitischen », in : Marta Kopji, Wojciech Kunicki (dir.), Nietzsche und Schopenhauer : Rezeptionsphänomene der Wendezeiten, Leipzig, Leipziger Universitätsverlag, 2006, p. 81-102, ici p. 83 . 
cette vision du monde. Dans la première période de l'activité littéraire de Thomas Mann - entre 1893 et 1912 - les personnages juifs ou décrits par des traits physiques ou de caractère qui leur sont traditionnellement associés, obéissent à des stéréotypes de son époque, qui caractérisent la culture de l'élite intellectuelle d'alors : ainsi il s'agit la plupart du temps de parvenus, de commerçants aux traits orientaux et/ou exotiques. Ainsi trois nouvelles consacrent la majeure partie de leur diégèse à ces personnages qui sont moteurs de l'action : Dans La Volonté de bonheur, Les Enfants de Wotan et Gladius dei, le narrateur ne cache pas l'identité de ses personnages ; leurs noms sont immédiatement clairement connotés : le baron von Stein, Aarenhold ou le galeriste Blüthenzweig. La description physique des personnages s'apparente d'ailleurs aux clichés de l'époque concernant les juifs : les hommes sont la plupart du temps malingres et hirsutes et les femmes également peu gâtées par la nature sont ostentatoirement ornées de bijoux nombreux et voyants ou hypersensuelles - nous renvoyons ici par exemple à la description du docteur Leander, de Siegmund Aarenhold ou de sa sœur ${ }^{34}$. Nous ne nous attarderons pas sur ce point ici étant donné qu'il existe quelques excellentes études sur la question : nous nous bornerons à rappeler simplement que malgré tout Thomas Mann s'inscrit dans son époque et que ces écrits, comme Altesse royale (Königliche Hoheit) (1906-1909) avec le personnage du docteur Sammet ou son essai intitulé Die Lösung der Judenfrage (1907), sont révélateurs de problèmes qui agitent toute la société 35 .

Le cas d'Amra dans Louisette est un cas intéressant : en effet elle s'approprie un prénom oriental - Amra signifie en arabe « vie, longévité »-en prenant les premières lettres de ses quatre prénoms :

Il y a trente ans, [...] elle avait reçu sur les fonts baptismaux les prénoms d'Anna Marguerite Rose Amélie ; mais par une juxtaposition de leurs diverses initiales, on ne l'appelait jamais qu'Amra, nom qui s'harmonisait comme pas un avec sa personnalité. En effet, si les ténèbres de sa chevelure épaisse et souple [...] étaient d'un simple brun de châtaigne, son épiderme d'un jaune mat et bistré tout à fait méridional recouvraient des formes qui semblaient, elles aussi, mûries au soleil du sud. Leur plénitude végétative et indolente évoquait celle d'une sultane.

34. - Romans et nouvelles II, Les enfants de Wotan, p. 40/Wälsungenblut, « Ein außerordentliches und fortwährendes Bedürfnis nach Reinigung », p. 440 ; ou encore «Sein Bartwuchs war so stark, daß er, wenn er abends ausging, genötigt war, sich ein zweitesmal davon zu säubern. », ibid., p. 441 (la pilosité est associée à la lubricité du personnage) ; ou la description du docteur Leander dans Tristan (version allemande, p. 163).

35. - Voir notamment l'ouvrage Manfred Dierks et Ruprecht Wimmer (dir.), Thomas Mann und das Judentum (Thomas-Mann-Studien, Bd. 30), Frankfurt a.M., Klostermann, 2004. 
Chacun de ses mouvements d'une nonchalance sensuelle confirmait d'ailleurs l'impression que chez elle le cœur primait la raison ${ }^{36}$.

Amra a donc choisi un prénom qui reflète et correspond à son physique méridional, c'est-à-dire « étranger », et le portrait est le parfait reflet du stéréotype non seulement de la femme fatale, mais aussi de celui de la femme orientale - la sultane - telle qu'on la trouve dans la littérature mais aussi l'opéra fin-de-siècle - que ce soit Salomé ou Lulu par exemple. Elle représente le type de la femme qui utilise ses charmes pour obtenir ce qu'elle désire et qui est en général cruelle et impitoyable ${ }^{37}$, et ce type s'inscrit dans le contexte de la rédaction et de la publication de l'ouvrage d'Otto Weininger, Geschlecht und Charakter (1903), qui oppose dans une lutte sans merci le principe masculin au principe féminin.

Pour conclure, nous pouvons affirmer que Thomas Mann produit d'un côté une identité culturelle univoque - les mariages mixtes sont en général malheureux, voire des échecs (dans Tonio Kröger, La Volonté de bonheur, Les Enfants de Wotan par exemple) - et hégémonique, marquée par le respect de l'ordre bourgeois et étatique strict. L'espace imaginaire de Thomas Mann établit ainsi au départ des frontières définies - frontières à l'origine du nationalisme mannien qui est produit par une vision particulière de l'Allemagne à l'époque (qui, elle, n'a rien d'exclusivement mannien par contre) et qui va reléguer les éléments considérés comme non allemands dans les marges du récit, voire les en exclure tout simplement par leur disparition de la diégèse. Certains personnages sont positionnés comme les « autres » de l'Allemagne et explicitement méprisés, tenus à distance par le narrateur. Ainsi, tous ceux qui ne correspondent pas à cette vision du Nord sont tout bonne-

36. - Romans et nouvelles I, Louisette, p. 95/Louischen, « Vor dreißig Jahren [...] war sie auf die Namen Anna, Margarethe, Rosa, Amalie getauft worden, aber man hatte sie [...] von jeher nicht anders als Amra genannt, ein Name, der mit seinem exotischen Klange zu ihrer Persönlichkeit paßte wie kein anderer. Denn obgleich die Dunkelheit ihres starken, weichen Haares [...] nur die Bräune des Kastanienkernes war, so zeigte ihre Haut doch ein vollkommen südliches mattes und dunkles Gelb, und diese Haut umspannte Formen, die ebenfalls von einer südlichen Sonne gereift erschienen und mit ihrer vegetativen und indolenten Üppigkeit an diejenigen einer Sultanin gemahnten. Mit diesem Eindruck, den jede ihrer begehrlich trägen Bewegungen hervorrief, stimmte durchaus überein, daß höchstwahrscheinlich ihr Verstand von Herzen untergeordnet war. », p. 160.

37. - Ulrich Weinzierl, « Die „besorgniserregende Frau“. Anmerkungen zu Luischen, Thomas Manns „peinlichster Novelle“ », in : Eckhard Heftrich, Hans Wysling (Hg.), Thomas Mann Jahrbuch, Band 4, Frankfurt a.M., Klostermann, 1991. p. 9-20 : «Sie verkörpert den reinen, von keinerlei Geistigkeit angekränkelten Trieb, ist die leibhaftige Geschlechtlichkeit, und in solchem Fall, suggeriert uns der leicht erschauernde Erzähler, ist allemal Vorsicht am Platz. Allein, sie nützt nichts. », p. 14. 
ment « évincés » de la diégèse, soit ils disparaissent du récit sans qu'une explication en soit donnée, soit plus régulièrement ils sont frappés par la maladie, la déchéance et la mort.

Mais de l'autre, dans une vision postcoloniale, il ne faut pas oublier de mentionner que dans les récits de jeunesse, qui sont en grande partie aussi autobiographiques, les personnages des villes hanséatiques, comme nous l'avons rappelé, profitent de l'exploitation par l'Europe des autres continents, mais sans que cela ne soit jamais ouvertement thématisé. Le monde de Thomas Mann vit de cette exploitation à tous les sens du terme. Mais elle est présentée de manière indirecte, biaisée par le narrateur : en effet celui-ci insiste sur le métissage, le croisement entre le Nord et le Sud, comme dans le cas personnel de l'auteur. Ainsi dans Tonio Kröger, le narrateur semble se mettre du côté des opprimés de la « vision coloniale », tandis que dans La Volonté de bonheur, par exemple, il semble très ouvertement endosser les préjugés antisémites de l'élite intellectuelle de l'époque ; par contre dans Les Enfants de Wotan la fin est ambiguë et pourrait être interprétée comme une victoire de ces personnages considérés comme marginaux :

- Mais Beckerath... murmura-t-elle en cherchant à mettre de l'ordre dans ses pensées. - Beckerath, Gigi... Qu'est-ce qu'il devient dans tout cela?

- Dans tout cela ?... répéta-t-il.

Et il ajouta, cependant que les signes de sa race apparaissaient sur son visage avec une force éclatante : Eh bien, crois-tu que nous l'avons roulé, le goy !38

Cette fin qui ne permet pas de trancher et de définir clairement la position du narrateur est révélatrice de la situation dans laquelle se trouve également l'auteur : il sent, perçoit les fissures, les ruptures dans la vision du monde de ses contemporains et tente d'en donner une image, mais aussi de donner aussi une voix aux personnes considérées comme autres, marginales. Dans ses textes, Thomas Mann s'interroge, en fictionnalisant le problème sur la question fondamentale pour lui de l'altérité et plus précisément de l'hybridité. Ce sujet d'interrogation est peut-être ce qui fait la marque de ses nouvelles de jeunesse.

38. - Romans et nouvelles, II, Les Enfants de Wotan, p. 57/Wälsungenblut : "„Aber Beckerath..." sagte sie und suchte ihre Gedanken zu ordnen. Beckerath, Gigi... was ist nun mit ihm ?...

„Nun“, sagte er, und einen Augenblick traten die Merkzeichen seiner Art sehr scharf auf seinem Gesichte hervor, ,was wird mit ihm sein? Beganeft haben wir ihn, - den Goy.“», p. 463. 
